\title{
Worse than Ever
}

National Cancer Institute

\section{Source}

National Cancer Institute. Worse than Ever. NCI Thesaurus. Code C121373.

In the all-time most severe or unfavorable condition. 